Provided for non-commercial research and educational use only. Not for reproduction or distribution or commercial use.

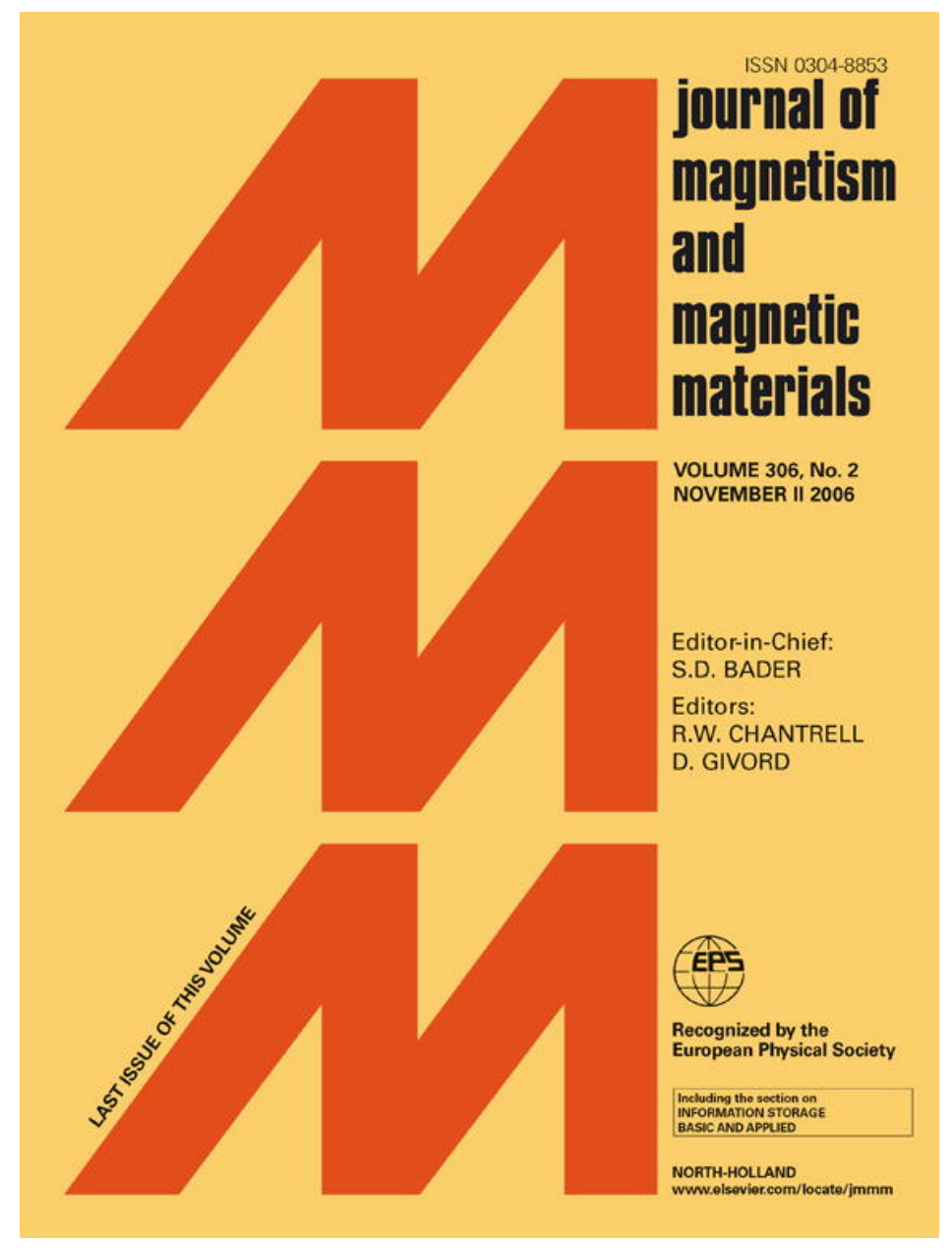

This article was originally published in a journal published by Elsevier, and the attached copy is provided by Elsevier for the author's benefit and for the benefit of the author's institution, for non-commercial research and educational use including without limitation use in instruction at your institution, sending it to specific colleagues that you know, and providing a copy to your institution's administrator.

All other uses, reproduction and distribution, including without limitation commercial reprints, selling or licensing copies or access,

or posting on open internet sites, your personal or institution's website or repository, are prohibited. For exceptions, permission may be sought for such use through Elsevier's permissions site at: 


\title{
Magnonics: Experiment to prove the concept
}

\author{
V.V. Kruglyak*, R.J. Hicken \\ School of Physics, University of Exeter, Stocker Road, Exeter, EX4 4QL, UK
}

Received 6 December 2005; received in revised form 17 February 2006

Available online 24 March 2006

\begin{abstract}
An experimental scheme for studying spin wave propagation across thin magnetic film samples is proposed. The scheme is based upon the creation of picosecond pulses of strongly localized effective magnetic field via ultrafast optical irradiation of a specially deposited exchange bias or exchange spring layer. The spin waves are excited near the irradiated surface before propagating across the thickness of the sample. They are then detected near the other surface either within the finite optical skin depth using the linear magneto-optical Kerr effect in metallic samples or by the magnetic second harmonic generation. The experiment can facilitate investigations of propagating spin waves with wavelengths down to several nanometers and frequencies in excess of hundreds of Gigahertz. An experiment upon a periodically layered nanowire (a finite cross-section magnonic crystal) is numerically simulated, although the sample might equally well be a continuous film or an array of elements (e.g. nanowires) that either have uniform composition or are periodically layered as in a magnonic crystal. The experiments could be extended to study domain wall-induced spin wave phase shifts and can be used for the creation of spin wave magnetic logic devices.
\end{abstract}

(C) 2006 Elsevier B.V. All rights reserved.

Keywords: Superlattice; Periodic; Time-domain; Pump-probe; Exchange

Following recent developments in the experimental techniques applicable to magnetic systems, the idea of magnonic devices, in which spin waves (magnons) could be used for transmitting and processing information, has received an increased attention in the magnetics community [1-22]. Standing spin waves have been observed in micron sized thin-film metallic elements by means of the time-resolved scanning Kerr microscopy (TRSKM) and Brillouin light scattering (BLS) $[11,17]$. The propagation of spin waves within much larger ferrite films, that are either continuous or periodically structured in the film plane, has been investigated using the space and time-resolved BLS and inductive detection techniques $[7,9,10,13,19,20]$. It has been proposed that spin waves can be used to investigate domain wall properties within an Aharonov-Bohm geometry [14], and in magnetic logic devices [20]. Typically, the spin waves considered in those works had wavelengths of several micrometers and frequencies within the sub-ten Gigahertz range, and were of magneto-dipole rather than

\footnotetext{
*Corresponding author. Tel.: + 441392 264163; fax: + 441392264111.

E-mail address: V.V.Kruglyak@exeter.ac.uk (V.V. Kruglyak).
}

exchange character. At the same time, exchange spin waves have wavelengths of just a few nanometers and frequencies of tens of Gigahertz, and hence could be used in much faster devices of nanometer size. While exchange spin waves propagating perpendicular to the surface of thick ferrite films have been studied in the frequency domain in Ref. [7], a time domain experiment is required to take advantage of effects such as those predicted in Ref. [14].

In this paper, we propose an all-optical time domain experiment for investigation of propagating exchange spin waves in nanoscale metallic samples. We describe how spin waves with wavelengths of down to ten nanometers may be excited and detected, and how the frequency of the excitation may be controlled. We use the object oriented micromagnetic framework (OOMMF) [23] to model the signal resulting from the spin wave propagation along a periodically layered nanowire $[16,18]$.

The principle of the proposed experiment is shown in Fig. 1. Spin waves excited on the front surface of the sample propagate across its thickness and are detected on the rear surface. The excitation of short wavelength spin waves requires magnetic field pulses localized within a 


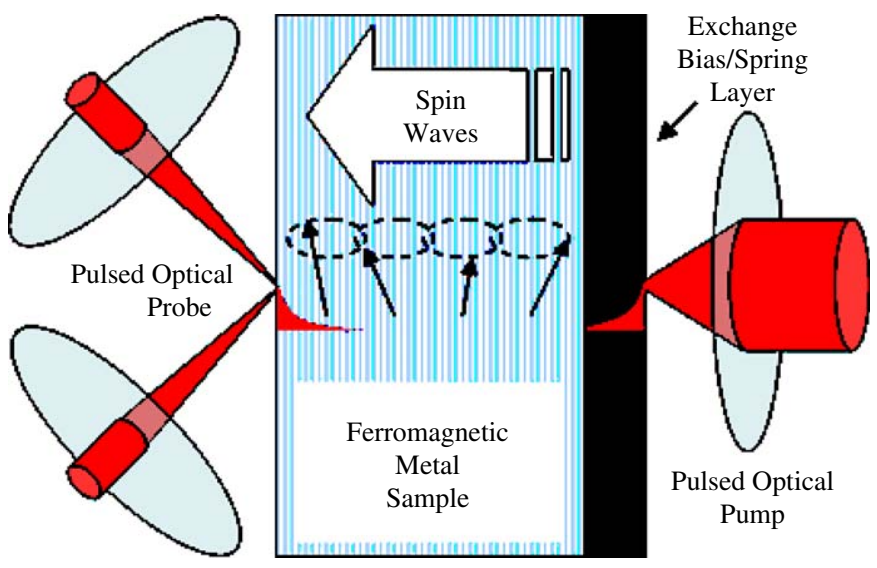

Fig. 1. A schematic of the proposed experiment is shown. The exchange bias/spring layer on the right-hand side is irradiated by the pump laser pulse, and hence a pulsed torque is exerted upon the adjacent thin-film multilayer sample, which also may be an array of uniform or multilayered elements. The torque excites spin waves that propagate towards the lefthand side surface of the sample where they are detected optically.

nanoscale region of the sample. For this purpose, an antiferromagnetic exchange bias layer is deposited onto the front surface of the sample. When irradiated by an intense femtosecond laser pulse, this layer exerts a pulsed effective magnetic field upon the adjacent ferromagnet [24]. The newly demonstrated optical excitation of an ultrafast antiferromagnetic to ferromagnetic phase transition in exchange spring materials represents another way of generating short localized effective field pulses [25]. In each case, the effective field pulses originate from the exchange interaction and hence are localized in the few monolayer thick interface region of the sample. Note that, in principle, magnetic field pulses with duration of hundreds of femtoseconds could be realized by the photo-inductive method proposed in Ref. [26]. However, the magnetic field pulses are not sufficiently localized to excite exchange-dominated spin waves. To study propagating rather than standing spin waves, the sample should be thick when compared to the depth of localization of the transient effective field, although thin when compared to the spin wave decay length, so that the signal detected on the rear surface is of measurable amplitude. The sample may be either a continuous film or an array of elements (e.g. nanowires), that has either uniform composition or is inhomogeneous (e.g. periodically layered as in the case of a magnonic crystal [18]). TRSKM or time-resolved BLS could be used to detect the transmitted spin waves. It is essential that the sample be metallic, so that the optical field is confined to the surface region. Then the magnetic response is sampled within the optical skin depth, i.e. about $10 \mathrm{~nm}$, which provides the lower limit for the spin wave wavelength detectable with this technique. The wavelength sensitivity can be further enhanced, virtually down to the atomic length, if magnetic second harmonic generation (MSHG) is used for detection.

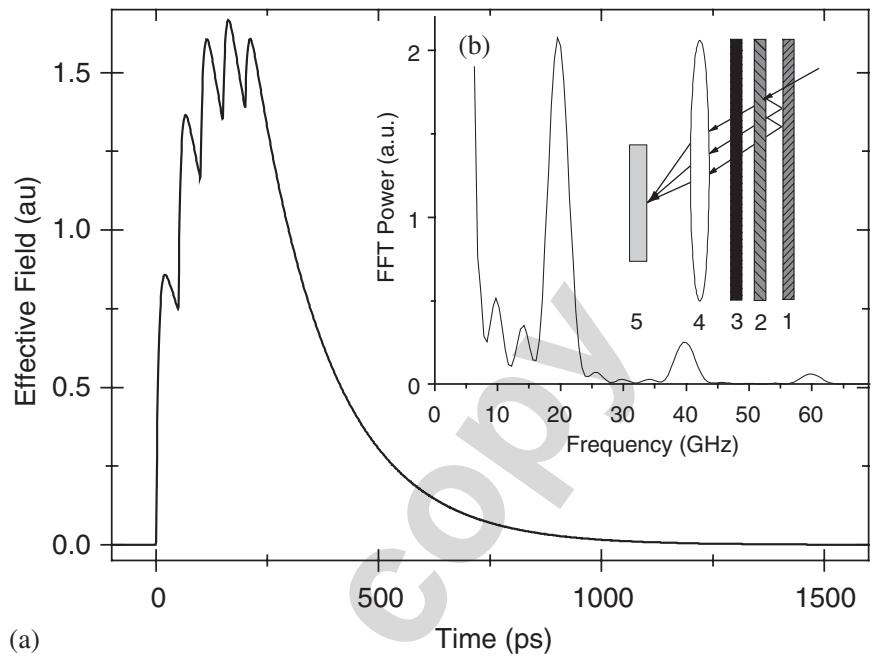

Fig. 2. (a) An exchange bias field pulse is shown that was obtained by a superposition of five pulses with rise time of $20 \mathrm{ps}$ and decay time of $170 \mathrm{ps}$ each. The subsequent pulses were delayed by $50 \mathrm{ps}$. (b) The Fourier spectrum of the pulse in (a) is shown. The inset shows the Fabry-Perotlike assembly used to form the optical pump pulse train leading to a superposition of three exchange bias field pulses. (1) is a mirror; (2) is a $10 / 90$ beam splitter; (3) is a gradient neutral density filter; (4) is a lens; (5) is the sample.

A single optical pump pulse may excite many modes with frequencies corresponding to the Fourier spectrum of the optically generated effective field pulse. For some applications, spin waves with a certain frequency may be preferred. As shown in Fig. 2, a combination of a Fabry-Perot etalon like assembly and a filter of varying optical density can be used to form an optical pulse train. The latter leads to an effective field pulse with a Fourier spectrum that contains a strong peak at a frequency corresponding to the time delay between subsequent optical pump pulses.

To explore what kind of information can be obtained from the proposed experiment, we have considered a $1 \mu \mathrm{m}$ thick film representing an array of periodically layered nanowires embedded into a nonmagnetic matrix with an exchange bias layer deposited on one surface. The nanowires lie perpendicular to the film surface and have a square cross-section with $20 \mathrm{~nm}$ side. Each nanowire consists of two different alternating homogeneous ferromagnetic layers with thickness of $20 \mathrm{~nm}$, and hence has a period of $40 \mathrm{~nm}$. The two layers have an exchange parameter of $13 \mathrm{pJ} / \mathrm{m}$, a uniaxial anisotropy of $0.5 \mathrm{~kJ} / \mathrm{m}^{3}$, a Gilbert damping parameter of 0.01 , and a $g$-factor of 2.1 , while they have different saturation magnetization values of 1 and $0.9 \mathrm{MA} / \mathrm{m}$. The external bias magnetic field of $0.01 \mathrm{~T}$ and the easy anisotropy axis are aligned parallel to the axis of the nanowire. The interfaces are assumed to be sharp and flat, and lie perpendicular to the easy axis. The nanowires are assumed to be sufficiently separated that the inter-wire dipolar interaction can be neglected, and so we have performed numerical simulations for an isolated nanowire only. The exchange bias layer is assumed to have 
identical magnetic properties as those studied in Ref. [24]. Therefore we have assumed that, upon excitation by a picosecond laser pulse, a pulsed exchange bias field of $1 \mathrm{mT}$ amplitude with rise time of $20 \mathrm{ps}$ and decay time of $170 \mathrm{ps}$ is created in the $2 \mathrm{~nm}$-thick end region of the nanowire adjacent to the exchange bias layer. The simulations were performed with cells of $0.5 \times 20 \times 20 \mathrm{~nm}^{3}$ size, and the magnetic state of the nanowire was recorded every $5 \mathrm{ps}$ within a period of $5 \mathrm{~ns}$ after the excitation.

The results of simulations are presented in Fig. 3. On the "time-distance" plot, the propagation of the perturbation at the left end of the wire is first seen in the form of almost straight beams of propagating spin waves slightly distorted due to the interference with reflections from interlayer interfaces. The shortest wavelength modes reach the right end in less than $200 \mathrm{ps}$, while the main portion of spin waves arrives after about 700 ps. After that a characteristic "grating" pattern of interference between the forward modes and those reflected from the right wire end is formed. A mode of smaller frequency as compared to that of the propagating spin waves is confined near the wire end by the nonuniform demagnetizing field [27], and serves as a

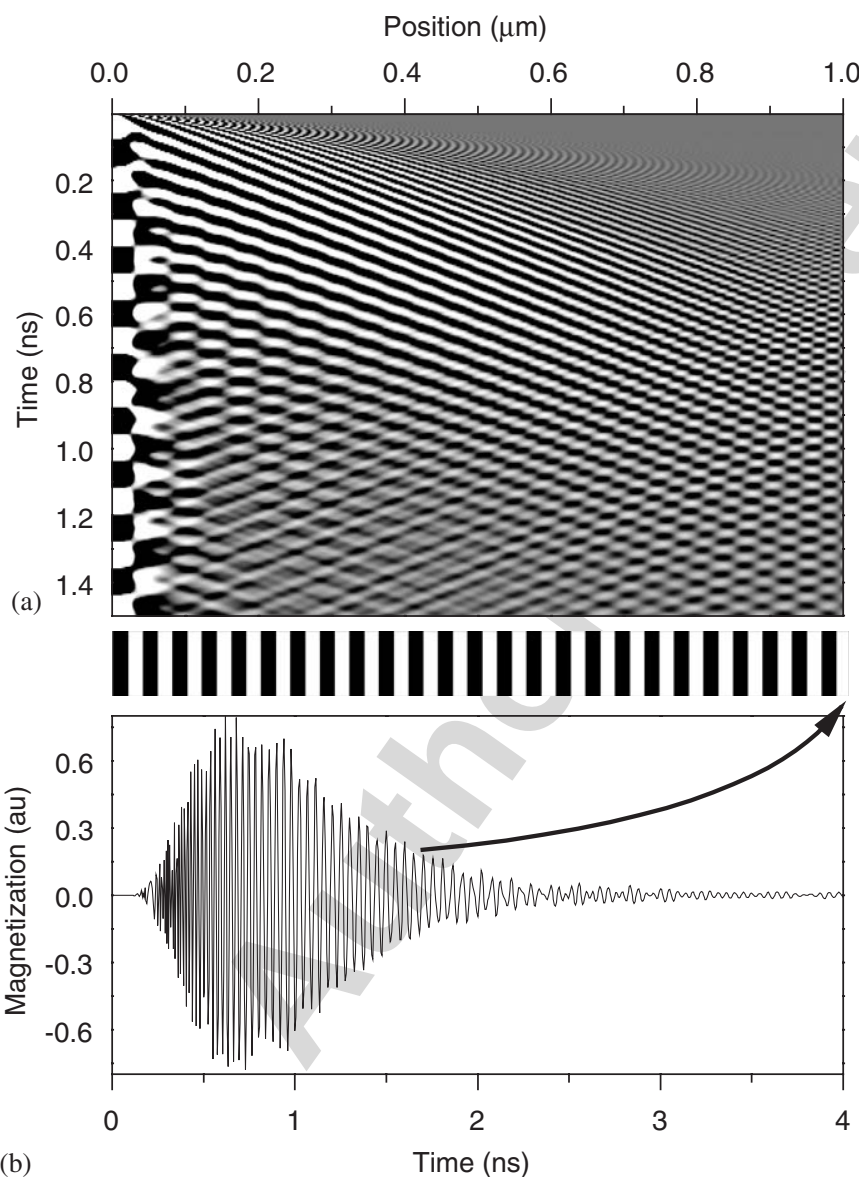

Fig. 3. (a) The results of the simulations are shown on the "timedistance" plane. The grey scale represents the magnetization component perpendicular both to the nanowire axis and the pulsed effective field. (b) The time-resolved signal obtained by averaging the response of the last (white) layer of the nanowire is shown. source of propagating modes. Fig. 3(b) shows a timeresolved signal obtained by averaging the response of the last layer of the nanowire, i.e. similar to the signal measured in the proposed experiment if the skin depth of the probe is about $20 \mathrm{~nm}$.

Fig. 4(a) shows a two-dimensional fast Fourier transform of the "time-distance" data array, a portion of which is presented in Fig. 3(a). One can immediately identify the parabolic dispersion relation of the exchange-dominated spin waves. Branching is observed at the points where the inverse wavelength is equal to $12.5 i / \mu \mathrm{m}$ ( $i$ is integer), i.e. at the Brillouin zone boundaries [18]. Fig. 4(b) shows Fourier spectra calculated from time-resolved signals obtained from spatial averages of different portions of the nanowire. Within the continuous spectrum of propagating spin waves, one can see clear dips that correspond to the band gaps at the Brillouin zone boundaries [18,28]. We note that the spectra from the last layer and the last cell (of the simulation) of the wire are similar to those which could be observed experimentally using TRSKM and MSHG, respectively.
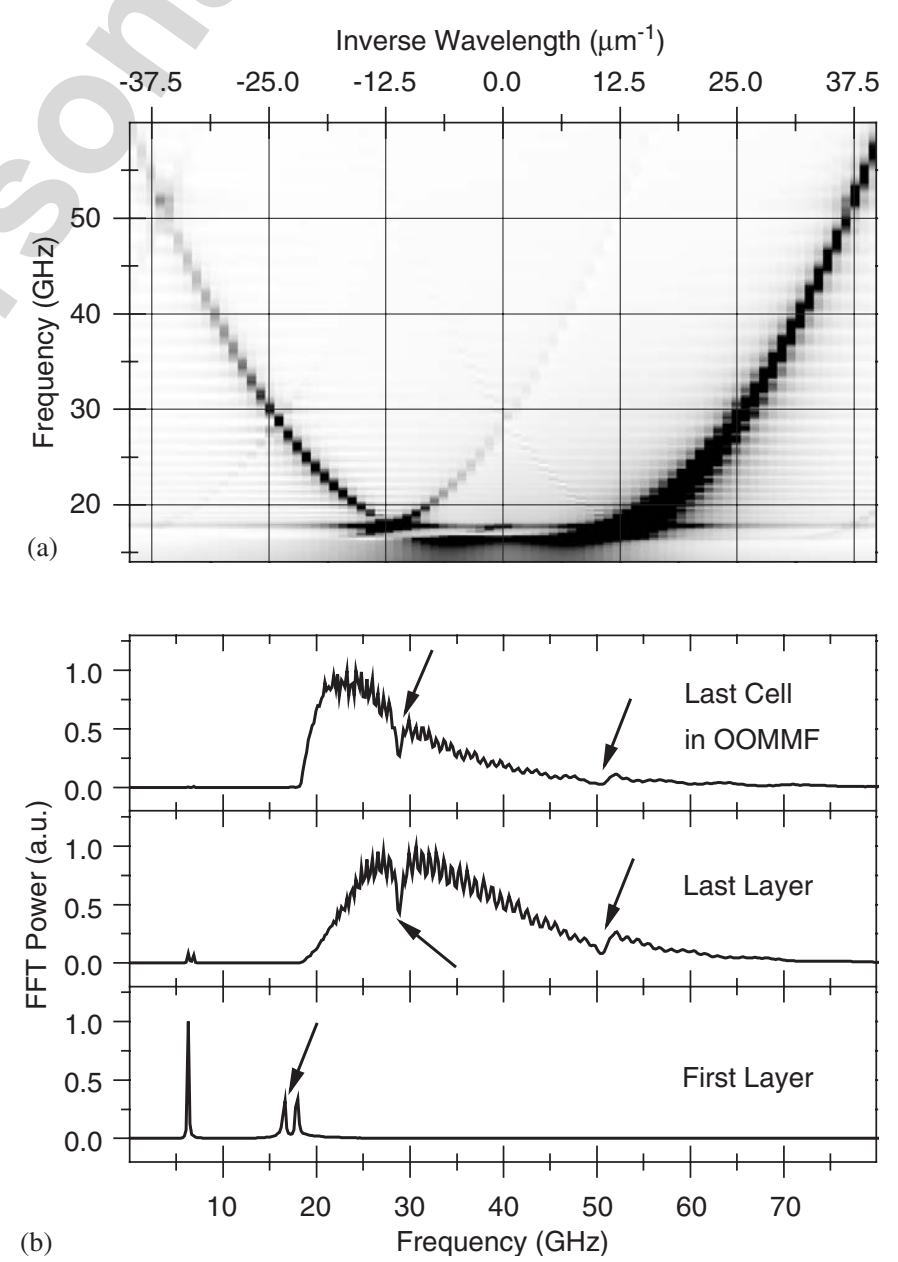

Fig. 4. (a) The two-dimensional fast Fourier transform of the "timedistance" data array from Fig. 3(a) is shown. (b) Fourier spectra calculated from average time-resolved signals from the last cell (of the OOMMF simulations), the last layer, and the first layer of the nanowire are presented in the top, middle, and bottom panels, respectively. The arrows mark positions of the band gaps. 
In summary, we have proposed an experimental scheme for studying spin wave propagation across thin film samples. We have described how short-wavelengths spin waves may be excited and detected, and how the frequency of excited spin waves may be controlled within the Gigahertz regime. While micromagnetic simulations have been presented for experiments performed upon a periodically layered nanowire, the sample might equally well be a continuous film or an array of elements (e.g. nanowires) that either have uniform composition or are periodically layered as in a magnonic crystal. The experiments could be extended to study domain wall-induced phase shifts within the Aharonov-Bohm geometry proposed in Ref. [14], and constitute the basis for realization of the spin wave magnetic logic devices proposed in Ref. [20].

The authors acknowledge the financial support of the UK Engineering and Physical Sciences Research Council (EPSRC) and the New Energy and Industrial Technology Development Organization (NEDO).

\section{References}

[1] C.G. Sykes, J.D. Adam, J.H. Collins, Appl. Phys. Lett. 29 (1976) 388.

[2] Y.V. Gulayev, S.A. Nikitov, V.P. Plesskii, Fiz. Tverd. Tela (Leningrad) 22 (1980) 2831.

[3] P. Grünberg, K. Mika, Phys. Rev. B 27 (1983) 2955.

[4] R.E. Camley, T.S. Rahman, D.L. Mills, Phys. Rev. B 27 (1983) 261.

[5] N.N. Chen, A.N. Slavin, M.G. Cottam, Phys. Rev. B 47 (1993) 8667.

[6] K.Y. Guslienko, Fiz. Tverd. Tela (St. Petersburg) 37 (1995) 1603 [Phys. Solid State 37 (1995) 870].

[7] P.E. Zilberman, A.G. Temiryazev, M.P. Tikhomirova, Usp. Fiz. Nauk 165 (1995) 1219.

[8] S.A. Nikitov, P. Tailhades, C.S. Tsai, J. Magn. Magn. Mater. 236 (2001) 320 .
[9] G.A. Melkov, Y.V. Kobljanskyj, A.A. Serga, V.S. Tiberkevich, A.N. Slavin, Phys. Rev. Lett. 86 (2001) 4918.

[10] Y.V. Kobljanskyj, G.A. Melkov, V.S. Tiberkevich, V.I. Vasyuchka, A.N. Slavin, J. Appl. Phys. 93 (2003) 8594.

[11] A. Barman, V.V. Kruglyak, R.J. Hicken, A. Kundrotaite, M. Rahman, Appl. Phys. Lett. 82 (2003) 3065.

[12] H. Puszkarski, M. Krawczyk, Solid State Phen. 94 (2003) 125.

[13] S.O. Demokritov, A.A. Serga, A. Andre, V.E. Demidov, M.P. Kostylev, B. Hillebrands, A.N. Slavin, Phys. Rev. Lett. 93 (2004) 047201.

[14] R. Hertel, W. Wulfhekel, J. Kirschner, Phys. Rev. Lett. 93 (2004) 257202.

[15] V.V. Kruglyak, A.N. Kuchko, J. Magn. Magn. Mater. 272-276 (2004) 302.

[16] V.V. Kruglyak, A.M. Kuchko, V.Y. Gorobets, Metallofiz. Noveish. Tekhn. 26 (2004) 579.

[17] K. Perzlmaier, M. Buess, C.H. Back, V.E. Demidov, B. Hillebrands, S.O. Demokritov, Phys. Rev. Lett. 94 (2005) 57202.

[18] V.V. Kruglyak, R.J. Hicken, A.N. Kuchko, V.Y. Gorobets, J. Appl. Phys. 98 (2005) 014304.

[19] S.L. Vysotskii, S.A. Nikitov, Y.A. Filimonov, Zh. Eksp. Teor. Fiz. 128 (2005) 636 [J. Exp. Theor. Phys. 101 (2005) 547].

[20] M.P. Kostylev, A.A. Serga, T. Schneider, B. Leven, B. Hillebrands, Appl. Phys. Lett. 87 (2005) 153501.

[21] K.-S. Lee, S. Choi, S. Kim, Appl. Phys. Lett. 87 (2005) 192502.

[22] A.N. Kuchko, M.L. Sokolovskii, V.V. Kruglyak, Physica B 370 (2005) 73.

[23] M. Donahue, D.G. Porter, OOMMF User's guide, Version 1.0, Interagency Report NISTIR 6376, NIST, Gaithersburg, MD, 1999, URL: http://math.nist.gov/oommf

[24] M.C. Weber, H. Nembach, J. Fassbender, J. Appl. Phys. 95 (2004) 6613.

[25] J.U. Thiele, M. Buess, C.H. Back, Appl. Phys. Lett. 85 (2004) 2857.

[26] V.V. Kruglyak, M.E. Portnoi, Pis'ma Zh. Tekhn. Fiz. 31 (2005) 20 [Techn. Phys. Lett. 31 (2005) 1047].

[27] V.V. Kruglyak, A. Barman, R.J. Hicken, J.R. Childress, J.A. Katine, Phys. Rev. B 71 (2005) 220409(R).

[28] The dips were absent in similar simulations performed for a uniform sample (not shown). 